\title{
Study of the Ph of Saliva and Dental Plaque Using Dental Preparations Containing Basil and Eucalyptus Essential Oils
}

\author{
Silviya Dimitrova ${ }^{1}$, Dimitar Dimitrov $^{2}$, Elena Boyadzhieva ${ }^{3}$, \\ Emiliya Simeonova ${ }^{4}$ \\ ${ }^{1}$ Assistent Professor, Department Of Operative Dentistry And Endodontics, Faculty Of Dental Medicine, \\ Medical University- Plovdiv, Bulgaria \\ ${ }^{2}$ Associate Professor, Department Of Technology Of Tobacco, Sugar, Vegetable And Essential Oils, \\ Technological Faculty, University Of Food Technologies- Plovdiv, Bulgaria \\ ${ }^{3}$ Assistent Professor, Department Of Operative Dentistry And Endodontics, Faculty Of Dental Medicine, \\ Medical University-Plovdiv, Bulgaria \\ ${ }^{4}$ Assistent Professor, Department Of Operative Dentistry And Endodontics, Faculty Of Dental Medicine, \\ Medical University-Plovdiv, Bulgaria
}

\begin{abstract}
The prevention of dental caries searches for possibilities to control biomarkers, such as $p H$ of saliva and dental plaque, with the use of natural products. The objective is to determine the effect of two dental preparations containing basil and eucalyptus essential oils, on the saliva and plaque pH. Preparation H3 contains hydrophilic vehicle from polyethylene glycols, preparation L3 contains hydrophobic vehicle from silicon dioxide. The study includes 90 individuals, distributed in three groups - control, H3-treated and L3treated. The applications are done on days 1, 3, 5, 7 and 9. The saliva $p H$ is tested with Saliva Check Buffer $(G S)$, and the dental plaque $\mathrm{pH}$ with Plaque indicator kit (GS). The time control periods are day 0, day 10 and 1 month.

The statistical processing is done with Fisher's Exact Test, Mann-Whitney U test, Wilcoxon signed-rank test, Ms Excel 2010 and $R$-Statistics, graphic visualization with bar-charts.

The use of dental preparation $\mathrm{H} 3$ and $L 3$ results in reduction of the cases with critically low $\mathrm{pH}$ in the saliva and dental plaque, more markedly with L3. Statistically significant is the effect between day 0 and day 10, and there is a tendency of maintaining the values on month 1.
\end{abstract}

Keywords: biofilm, essential oils, prevention, saliva

\section{Introduction}

An important step in forming the prevention-oriented healing philosophy is the evaluation of the risk of disease, by analyzing the risk factors, and its individual management. The role of the bacterial biofilm, with activation of its pathogenic potential depending on the environmental factors, has been categorically proven in the etiopathogenesis of primary and secondary dental caries. Low values of saliva and plaque $\mathrm{pH}$ could prevent the development of some oral microorganisms, but at the same time change the microbial ecology by stimulating the proliferation of acidogenic and aciduric microbial species. There is a proven correlation between the presence of Streptococcus mutans and Lactobacillus in the saliva and dental plaque. A significant moment in the modern antimicrobial therapy is the medicinal resistance of the microorganisms. The dental medicine uses mostly antibiotics and chemotherapeutics with proven efficiency. Their disadvantages are related to the synthetic origin, non-volatility, cumulating in the organism, selection of resistant strains microorganisms, causing genetic mutations in bacteria, etc. Their use is associated with different side effects and complications, such as allergies, candidiases, reduced immunity, which not always can be predicted.

\section{Literature review}

In the last years, there is a worldwide tendency of increased use of natural products, as a result of their proven pharmacological effect on the oral environment, as well as in relation with effective prevention of dental caries $[1,2,3]$. The essential oils have the ability to inhibit the plaque formation, as they demonstrate not only antimicrobial effect, provided mostly by the aromatic agents, but they also have deeper penetration in the biofilm, compared to other agents, such as stannous fluoride and triclosan $[4,5,6]$. There are many antimicrobial agents, most of them designed for home use, where there is no dosage control, frequency and intended use. Quite naturally, their efficiency is subject of discussion in many scientific reports $[4,5]$, as the general opinion is that they can be successfully used as supplementary, but not as primary agents for caries prophylactics. In relation with overcoming the microbial resistance, a number of authors comment on limitation the use of antimicrobial agents not only to high-risk patients, but also only to high-risk dentition areas $[4,5]$. 
Different biochemical and/ or bacteriological parameters of the saliva have been analyzed showing unsatisfactory correlation with the development of the carious process [7, 8, 9]. In order to program the caries activity, today are tested risk indexes and biomarkers, such as saliva flow, $\mathrm{pH}$, buffering capacity of saliva, plaque $\mathrm{pH}$, potassium and phosphorus concentration in saliva, salivary levels of Streptococcus mutans and Lactobacillus, dental morphology and diet habits, etc [10, 11, 12, 13]. Parameters of the dental plaque, with significant role in the initiation of dental caries have long been neglected, until recent studies show, that plaquefactors could be good predictors [14].

\section{Materials And Methods}

Clinical study has been conducted following approval of the Scientific Ethics Committee of Medical University Plovdiv. All participants are informed in detail about the nature of the study, and have given their written consent to participate in it. Object of the study are 2 dental preparations, designated as H3 - with hydrophilic vehicle and L3 - with hydrophobic vehicle, with the following composition: H3 - 40\% basil oil, $40 \%$ eucalyptus oil, $20 \%$ polyethylene glycols $\left(\mathrm{PEG}_{4000}\right)$; L3 - 46\% basil oil, $46 \%$ eucalyptus oil, $8 \% \mathrm{SiO}_{2}$ (Aerosil 200). The study includes 90 clinically healthy individuals, aged between 20 and 40 years, with at least 20 natural teeth, of which at least 5 obturated, with normal oral hygiene, diet and lifestyle. Patients with information for general diseases and allegories, taking medications for the period of the study and at least one month before that, with clinical signs of inflammatory processes in the parodontium and the oral cavity, were not included. The participants were equally distributed randomly in 3 groups, as follows: Group 1 - controls; Group 2 - treated with H3 dental preparation; Group 3 - treated with L3 dental preparation.Before the beginning of the study (day 0), the initial saliva and plaque $\mathrm{pH}$ values of all participants were registered, and professional hygiene was performed for removal of tartar and dental plaque. The participants were instructed to maintain personal oral hygiene using brush and toothpaste without active ingredients, morning and evening for 2 minutes, in usual way.

In Groups 2 and 3, the respective dental preparations were applied in thin even layer on all teeth using a small brush, on days 1, 3, 5, 7 and 9. The surfaces of the teeth were preliminary cleaned with hydrogen peroxide, then washed and dried. The isolation of the operating field was done with lignin rolls for the duration of the procedure, and 15 minutes after the applications. The participants were instructed not to eat and drink within 2 hours after the procedures. The saliva $\mathrm{pH}$ is tested with Saliva check buffer (GS), and the dental plaque pH with Plaque indicator kit (GS). The time control periods are day 0, day 10 and 1 month. The saliva and dental plaque samples are taken at least 2 hours after the oral-hygienic procedures. The collection of saliva is done within 5 minutes in sterile containers. Approximal dental plaque is taken for analysis with special test curettes. The change in the $\mathrm{pH}$ level is registered with change in the color of the indicators, registered according to the respective scales and tests, and photographed. Statistical hypothesis with exact calculation of $\mathrm{p}$-value are used. For the statistical difference between the values of a given parameter in the different study groups are used Kruskal Wallis and Mann-Whitney U nonparametric test (for continuous random variables in comparison of two or more than two groups simultaneously). The statistically significant change of the value of a parameter, as a time function in a group, is proved with McNemar and Marginal Homogeneity test. The graphic visualization is done with bar charts, nominated to $100 \%$, for representation of the percentage share for a given category of data. Ms Excel 2010 and R - Statistics software is used (www.r-project.org).

\section{Results}

The results from the study of the saliva $\mathrm{pH}$ on day 0 , day 1 and month 1 of the three controlled groups are shown in (Fig. 1), (Fig. 2) and (Fig. 3).

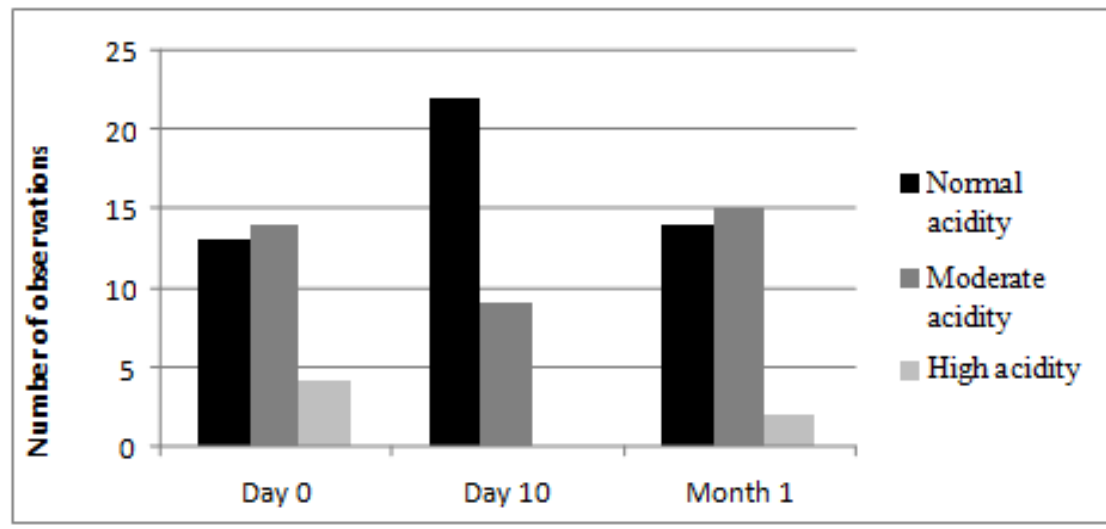

Figure 1. Results from the studied salivary $\mathrm{pH}$ in the group treated with product $\mathrm{L} 3$ in time 


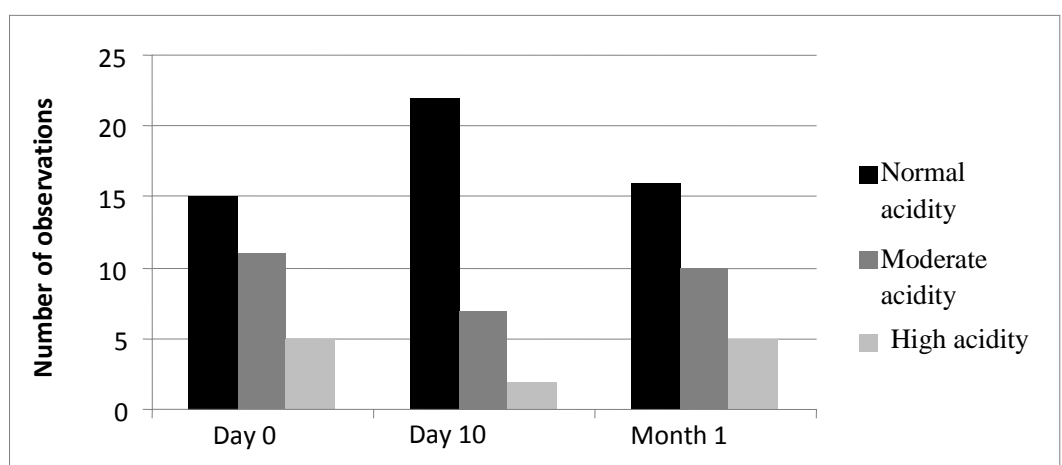

Figure 2. Results from the studied salivary $\mathrm{pH}$ in the control group in time

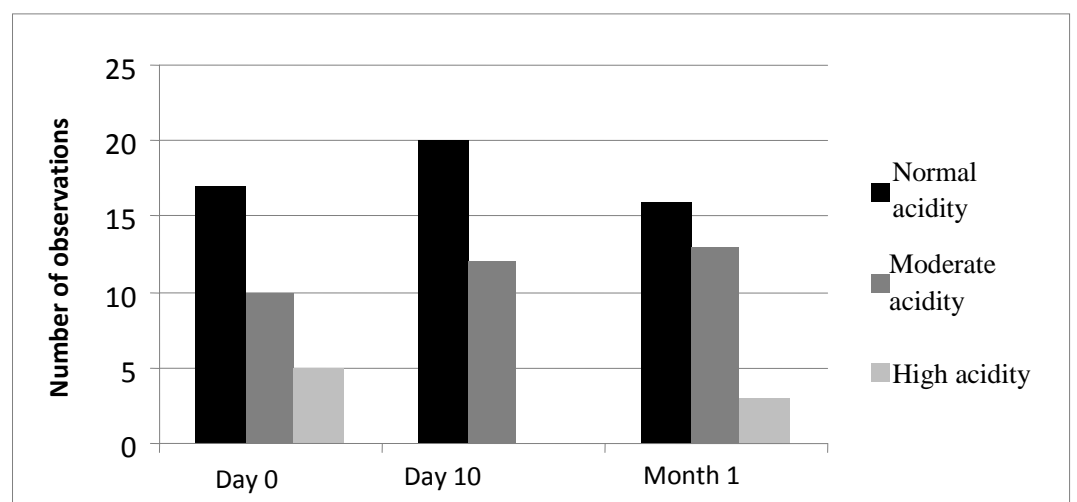

Figure 3. Results from the studied salivary $\mathrm{pH}$ in the group treated with product $\mathrm{H} 3$ in time

Statistical analysis of the results shows, that the treatment with H3 and L3 results in reduction of the cases of high acidity, which is most expressed and statistically significant between day 0 and day 10 ( $p$-value = 0,$011 ; p<0,05$ for group H3, and $p$-value $=3,11.10^{-4} ; p<0,0$ for group L3). The results in the control group show, that although there is decrease of the cases with high acidity on day 10, compared to day 0 (p-value $=0,004$; $\mathrm{p}<0,05$ ), after day 10 these cases increase again, and on month 1 they are close to day 0 ( $p$-value $=0,564$; $\mathrm{p}>0,05)$. The changes are most probably due to the reduction of the microbial levels of the tested cariogenic microorganisms. With H3 statistical difference is observed between day 0 and day 10 ( $\mathrm{p}$-value $=0,011 ; \mathrm{p}<$ $0,05)$, without significance between day 10 and month 1 ( $p$-value $=0,052 ; p>0,05)$, which shows that there is a permanent decrease of the acidity of the saliva after day 10. These results are quite expected with the use of preparation containing hydrophilic vehicle, as its effect on the salivary acid-producing microorganisms is expected to be high.

The results from the study of the $\mathrm{pH}$ of the dental plaque in time, are shown in (Fig. 4), (Fig. 5) and (Fig. 6), respectively for each of the tested groups.

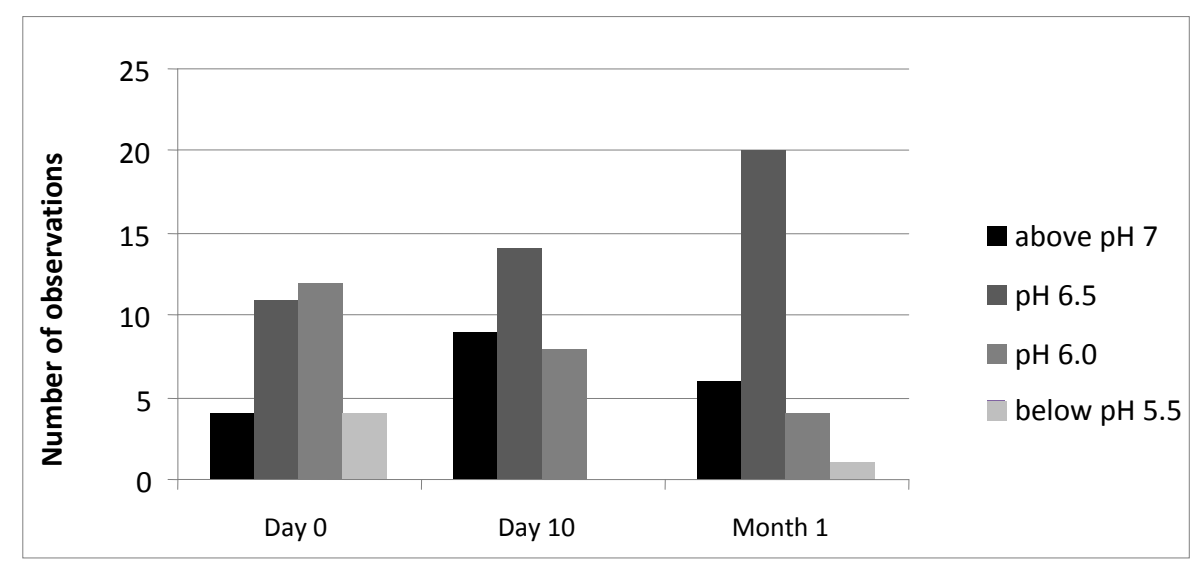

Figure 4. Results from the studied $\mathrm{pH}$ of the plaque in the group treated with product $\mathrm{L} 3$ in time 


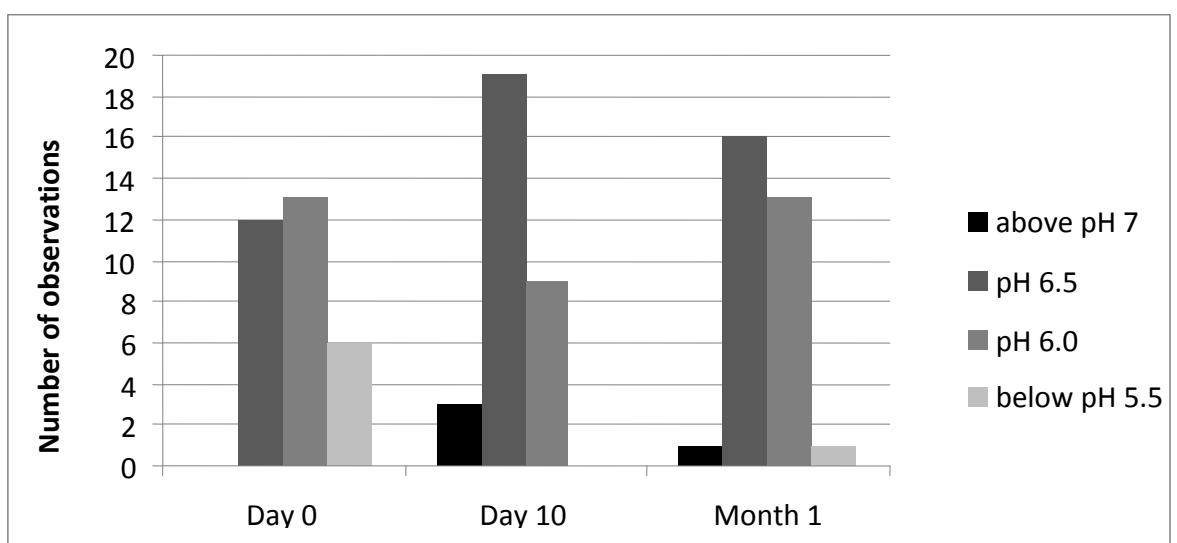

Figure 5. Results from the studied $\mathrm{pH}$ of the plaque in the control group in time

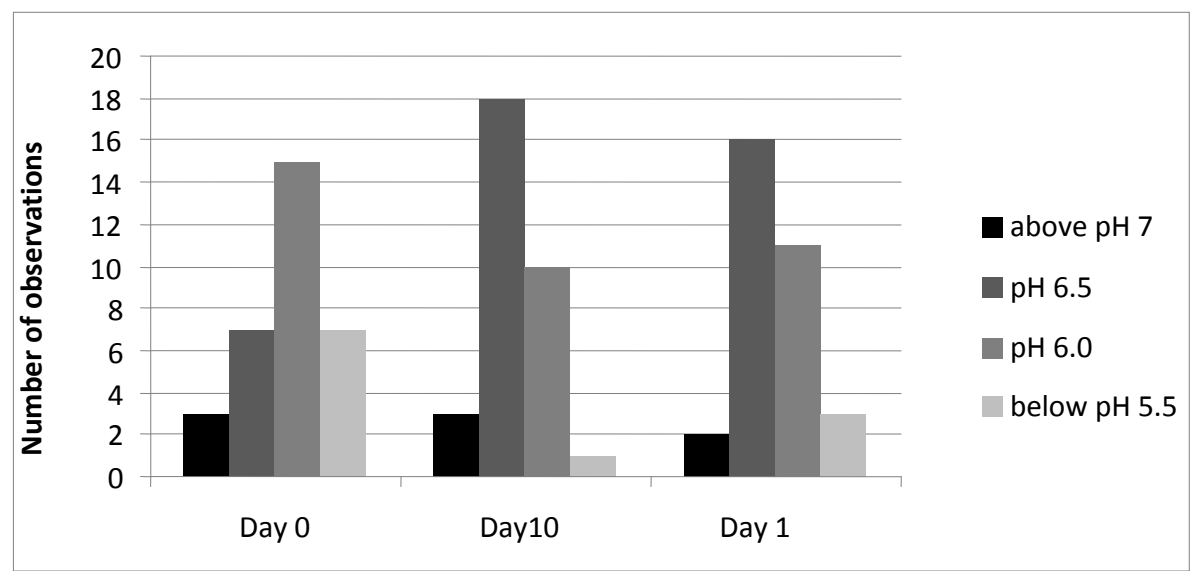

Figure 6. Results from the studied $\mathrm{pH}$ of the plaque in the group treated with product $\mathrm{H} 3$ in time

In the group treated with $\mathrm{H} 3$, there is an increase of the cases with $\mathrm{pH} 6.5$ and reduction of the cases with critical $\mathrm{pH}$ values below 5.5, as the difference is reliable between day 0 and day 10 ( $\mathrm{p}$-value $=3,74.10^{-5}$; $\mathrm{p}<0,05)$, and day 0 and month 1 ( $\mathrm{p}$-value $=0,008 ; \mathrm{p}<0,05)$. The obtained results are expected, as the acidity of the plaque is influenced by the activity and the metabolism of the microorganisms in the dental biofilm. The reduction of the dental plaque, tartar and microbial levels of Str. mutans and Lactobacillus, would quite logically bring similar change in the $\mathrm{pH}$ of the plaque. In the control group there is also increase of the $\mathrm{pH}$ values of the plaque, and on day 10 and month 1, there are $\mathrm{pH}$ values above 7.0, which are missing on day 0 . It is worth noting, that on day 10 the cases of $\mathrm{pH}<5.5$, which are evident in day 0 , disappear. It could be summarized, that the clinical oral prophylactics reduces the acidity of the plaque. In the group treated with L3 is observed the same tendency of increasing the $\mathrm{pH}$ values for all control periods, as at the same time there is a drastic decrease of the critical $\mathrm{pH}<5.5$ (Fig. 4). We should note, that on day 10 there are no cases of $\mathrm{pH}<5.5$, however, there is increase of the cases of $\mathrm{pH}>7.0$ ( $\mathrm{p}$-value $=3,74.10^{-5} ; \mathrm{p}<0.05$ ), as they appear again on month 1 , but the difference is insignificant ( $p$-value $=0.763 ; p>0.05$ ). The lack of significant difference between day 10 and month 1 ( $p$-value $=0.763 ; p>0.05$ ) in L3 shows, that there is permanent tendency towards reduction of the plaque acidity after day 10 . These results are expected for the preparation with hydrophobic vehicle (L3), considering the good adhesion to the dental surface and the prolonged release of the active ingredients. The increase of the plaque $\mathrm{pH}$ is most like due to the reduction of the acid-producing cariogenic microorganisms. At the same time, it is interesting, that we have similar results from the treatment with H3 (with hydrophilic vehicle) $(\mathrm{p}$-value $=0.083 ; \mathrm{p}>0.05$ between day 10 and month 1 ), although the lab results show its faster release in the saliva).

\section{Descussion}

Streptococcus mutans and Lactobacillus are known to be associated with the formation of white carious spots $[15,16]$, which are predictors of caries activity. During local changes, such as drop in the $\mathrm{pH}$ values and increased accumulation of biofilm, there is increased colonization of Streptococcus mutans, which create suitable acid environment for development of Lactobacillus. It is proven, that there is correlation between the presence of Str.muans and Lactobacillus in the saliva and in the dental plaque [14, 17]. The acidity of the 
saliva depends on the concentration of the acid-producing microorganisms - the higher it is, the lower the $\mathrm{pH}$ is, and respectively, with the increase of the saliva flow, the $\mathrm{pH}$ also increases [7, 17]. A previous study of the same preparations shows drastic decrease of the microbial levels of Streptococcus mutans and Lactobacillus on day 10. The obtained results are directly related to the reduction of the cases with low plaque pH level with both preparations, and confirm, that there is correlation between the amount of the acid-producing microorganisms in the saliva and the plaque. The registered reduction of the salivary microbial levels results in the reduction of the plaque levels, and respectively in increase of the plaque $\mathrm{pH}$ values.

Taking of samples for analysis of types of plaques, to study the caries activity, is commented by a number of authors [14]. Only 5\% of the lesions appear on the smooth bucco-lingual surface, and the fissure plaque, which is responsible for $85 \%$ of the lesions, is impossible to be collected in quantity sufficient for analysis $[14,18]$. In this study is used Saliva Check Buffer, and approximal plaque for testing the $\mathrm{pH}$ of the plaque. Our choice is based on the fact, that this is one of the riskiest zones for development of primary and secondary caries, and it is possible to collect sufficient quantity for analysis. Similarly, Carvalho R. et al. [19] study approximal plaque and also find it to be good choice. They comment that the location and the degree of its formation is different and corresponds to a number of other factors, such as diet and oral-hygiene habits, saliva flow rate, etc.

\section{Conclusion}

The use of dental preparations, with possible adhesion to the dental surfaces, slow washing by the saliva and prolonged release of the active ingredients, provides increasing of the $\mathrm{pH}$ values of the saliva and the dental plaque in immediate proximity to the risk zones.

\section{Future Scope}

The prevention and the non-operative treatment of the dental caries is risk patients with the use of antimicrobial agents for clinical topical application is part of the modern strategy for medical model and modern caries management.

\section{References}

[1]. JD. Featherstone, The science and practice of caries prevention. J Am Dent Assoc, 131(7), 2000, 887-899

[2]. MR. Pai, LD. Acharya, N. Udupa, Evaluation of antiplaque activity of Azadirachta indica leaf extract gel - a 6-week clinical study. J Ethnopharmacol, 90(1), 2004, 99-103.

[3]. G. Svensater, M. Borgstrom, GH. Bowden, S. Edwardsson, The acid-tolerant microbiota associated with plaque from initial caries and healthy tooth surfaces. Carres Res, 37(6), 2003, 395-403.

[4]. MA. Botelho, NAP. Nogueira, GM. Bastos, et al., Antimicrobial activity of the essential oil from Lippia sidoides, carvacrol and thymol against oral pathogens. Braz J Mcd Biol Res, 40(3), 2007, 349-356.

[5]. A. Dziedzic, R. Kubina, R.D. Wojtyczka, et al., The Antibacterial Effect of Ethanol Extract of Polish Propolis on Mutans Streptococci and Lactobacilli Isolated from Saliva, Hindawi Publishing Corporation, Evidence-Based Complementary and Alternative Medicine, 2013(2013), 2013, Article ID 681891, 12 pages.

[6]. I. Gera, The bacterial biofilm and the possibilities of chemical plaque control. Literature review. Fogorv Sz, 101(3), 2008, 91-99.

[7]. M. Marinova, Root caries. Comparative analysis of materials used during its treatment, doctoral diss., Medical University Sofia, Sofia, 2012.

[8]. WH. Bowen, Caries activity tests. Int Dent J, 19(2), 1969, 267-272.

[9]. WD. Miller, Microorganisms of the human mouth (Philadelphia: S.S. White Dental Mfg Co., 1890).

[10]. A. Bardow, JM ten. Cate, B. Nauntofte, B. Nyvad, Effect of unstimulated saliva flow rate on experimental root caries, Car Res, 37(3), 2003, 232-236.

[11]. G. Basil, BG. Bibby, RJ. Shem, Methods of caries prediction (Washington: Information Retrieval Inc, 1978).

[12]. M. Du, H. Jiang, B. Tat, et al., Root caries patterns and risk factors of middle-aged and elderly people in China. Community Dent Oral Epidemiol, 37(3), 2009, 260-266

[13]. S. Imazato, K. Ikebe, T. Nokubi, et al., Prevalence of root caries in a selected populaton of older adults in Japan, J Oral Rehab, 33(2), 2006, 137-143.

[14]. PI. Euan, YM. Dong, L. Yue, et al., Plaque minerals in the prediction of caries activity, Community Dent Oral Epidemiol, 30(1), 2002, 61-69.

[15]. M. Peneva, The dental caries during the $21^{\text {st }}$ century (Sofia: East-West, 2008).

[16]. S. Rimalovska, M. Kukleva, Secondary caries in temporary and permanent children's teeth- literature review - part 1, Dental medicine, 94(3), 2012, 204-212.

[17]. D. Karayasheva, M. Marinova, V. Dogandzhiyska, El. Boteva, Determining the salivary flow, buffer capacity and microbial numbers in active caries and caries resistant persons, Dental medicine, 90(1), 2008, 9-16.

[18]. RF. Wilson, FP. Ashley, The effects of experimental variations in dietary sugar intake and oral hygiene on the biochemical composition and $\mathrm{pH}$ of free smooth-surface and approximal plaque, J Dent Res, 67(6) 1988, 949-953.

[19]. R. Carvalho, VN. Tavares, S. Pereira, et al., Plaque and antigingivitis effect of lippia sidoides. A double-blind clinical study in humans, J Appl Oral Sci, 17(5), 2009, 404-407. 